\title{
Exotic nonrelativistic string
}

\author{
Roberto Casalbuoni* \\ Department of Physics, University of Florence, INFN, Florence, Italy \\ and Galileo Galilei Institute for Theoretical Physics, Florence, Italy \\ Joaquim Gomis ${ }^{\dagger}$ \\ PH-TH Division, CERN, CH -1211 Geneva 23, Switzerland \\ and Departament d'Estructura i Constituents de la Matèria, Facultat de Física, Universitat de Barcelona, \\ Diagonal 647, 08028 Barcelona, Spain \\ Giorgio Longhi ${ }^{\ddagger}$ \\ Department of Physics, University of Florence and INFN, Florence, Italy
}

(Received 8 October 2007; published 6 December 2007)

\begin{abstract}
We construct a classical nonrelativistic string model in $3+1$ dimensions. The model contains a spurion tensor field that is responsible for the noncommutative structure of the model. Under double-dimensional reduction the model reduces to the exotic nonrelativistic particle in $2+1$ dimensions.
\end{abstract}

DOI: 10.1103/PhysRevD.76.126004

PACS numbers: $11.25 . \mathrm{Mj}$

\section{INTRODUCTION}

The free massive nonrelativistic particle in $D$ dimensions has as a symmetry group, the Galilei group, with a central extension associated with the mass of the particle. Instead, in $2+1$ dimensions, there is a two-fold central extension [1-3], where the second central element is interpreted as a noncommutative parameter. These central extensions are related to a nontrivial Eilenberg-Chevalley cohomology of degree two. In fact every closed invariant 2 -form that locally is the differential of a 1 -form, which is not invariant, produces an extension of the algebra; see for example [4-6].

The exotic $(2+1) \mathrm{D}$ Galilei symmetry has appeared in the context of noncommutative geometry and condensed matter physics [7-18]. It is a symmetry of a free relativistic particle in a noncommutative plane in a special nonrelativistic limit $[9,11,12]$.

Instead, if one consider the nonrelativistic limit of a relativistic particle in the anti-de Sitter (AdS) space in $2+$ 1 dimensions, one finds a system with an exotic NewtonHooke symmetry [19]. The system has three essentially different phases [20], depending on the values of the two central charges which are present in the model. The subcritical and supercritical phases (describing 2D isotropic ordinary and exotic oscillators) are separated by the critical phase (one-mode oscillator).

Nonrelativistic extended objects have been recently considered in the literature. In particular, nonrelativistic string theory [21] (see also [22]) in flat space is a consistent decoupled sector of the bosonic string theory, whose world sheet, in the conformal field theory description [21], pos-

\footnotetext{
*casalbuoni@fi.infn.it

gomis@ecm.ub.es

†longhi@fi.infn.it
}

sesses the appropriate Galilean symmetry. Nonrelativistic string theory can be derived as a certain decoupling limit of the original relativistic theory, even though the theory can be written down without any reference to the original parent theory. The basic idea behind the decoupling limit is to take a particular nonrelativistic limit, in such a way that only states satisfying a Galilean invariant dispersion relation have finite energy, while the rest of the states decouple.

The action of nonrelativistic bosonic string theory, for a flat $D$ dimensional space-time, can be obtained with the method of nonlinear realizations [23] as a Wess-Zumino term of the appropriate (string) Galilean group [24,25]. The extended algebra has noncentral elements that transform nontrivially under the stability group.

Particle models can be obtained by a doubledimensional reduction of string models. It is natural to ask if it is possible to construct an exotic nonrelativistic string in $3+1$ dimensions, with other additional extended charges, such that by reduction one could obtain the exotic particle models appearing in the literature $[7,8,11,12]$. To this end we construct the classical action for exotic Galilean nonrelativistic strings, using the method of nonlinear realizations. We first construct the exotic string Galilei algebra in $3+1$ dimensions. This algebra contains new extra extended generators. As we will see, the parameters associated to these new extensions are related to noncommutative parameters. The string action is constructed from a 2-form, which is the product of two invariant Maurer-Cartan (MC) 1-forms of the exotic extended string Galilei algebra. The parameters of the string action are the tension and a tensor parameter (spurion), generalizing the magnetic field in the case of a particle.

We perform a classical Hamiltonian analysis of the model. The Hamiltonian turns out to be nonlocal in the original variables. If we introduce an appropriate change of 
variables, we can eliminate the nonlocality, ending with a theory formally equivalent to the ordinary nonrelativistic one for particular boundary conditions. Therefore, in this last case, the symmetry group of the model in the new variables is the nonexotic Galilei group.

The organization of the paper is as follows. In Sec. II we consider the extended exotic Galilei algebra. In Sec. III we introduce the string action, and in Sec. IV we study the dynamics in the static gauge, showing that there are second-class constraints in the model, then we proceed to evaluate the Dirac brackets. This analysis shows that the Dirac brackets of the position variables are not zero, giving rise to a noncommutative theory. In Sec. V we show that it is possible, through a change of variables, to make the model equivalent to the nonrelativistic string model, only for particular choices of the boundary conditions. We conclude with an outlook and a discussion in Sec. VI.

\section{EXOTIC EXTENDED GALILEI STRING ALGEBRA}

Let us consider a nonrelativistic string in a $D$ dimensional flat space-time.

We will denote by $x^{0}, x^{1}$, and $x^{2}, \ldots x^{D-1}$ the longitudinal (along the string) and transverse coordinates, respectively. The symmetry algebra of this system is the extended Galilei algebra, G [24,25], which is given by

$$
\begin{gathered}
{\left[P_{\mu}, P_{\nu}\right]=0, \quad\left[M_{\mu \nu}, P_{\rho}\right]=i\left(\eta_{\mu \rho} P_{\nu}-\eta_{\nu \rho} P_{\mu}\right),} \\
{\left[M_{\mu \nu}, M_{\rho \sigma}\right]=0,} \\
{\left[M_{a b}, P_{c}\right]=i\left(\eta_{a c} P_{b}-\eta_{b c} P_{a}\right),} \\
{\left[M_{a b}, M_{c d}\right]=i\left(\eta_{a c} M_{b d}+\eta_{b d} M_{a c}-\eta_{a d} M_{b c}-\eta_{b c} M_{a d}\right),}
\end{gathered}
$$

$$
\begin{gathered}
{\left[P_{\mu}, P_{a}\right]=0, \quad\left[M_{\mu a}, P_{\nu}\right]=i \eta_{\mu \nu} P_{a},} \\
{\left[M_{\mu \nu}, M_{\rho c}\right]=i\left(\eta_{\mu \rho} M_{\nu c}-\eta_{\nu \rho} M_{\mu c}\right),} \\
{\left[M_{\mu a}, M_{b c}\right]=-i\left(\eta_{a b} M_{\mu c}-\eta_{a c} M_{\mu b}\right),} \\
{\left[P_{a}, M_{\nu b}\right]=i \eta_{a b} Z_{\nu}, \quad\left[M_{\mu a}, M_{\nu b}\right]=i \eta_{a b} \epsilon_{\mu \nu} Z,}
\end{gathered}
$$

and

$$
\begin{gathered}
{\left[P_{\mu}, Z_{\nu}\right]=0, \quad\left[P_{\mu}, Z\right]=+i \eta_{\mu \nu} \epsilon^{\nu \lambda} Z_{\lambda},} \\
{\left[Z_{\mu}, M_{\rho \sigma}\right]=-i \eta_{\mu[\rho} Z_{\sigma]}, \quad\left[Z, M_{\rho \sigma}\right]=0 .}
\end{gathered}
$$

In these expressions $P_{\mu}, \mu=0,1$, are the longitudinal translation generators, $P_{a}, a=2, \ldots D-1$ are the transverse ones, $M_{\mu \nu}, M_{\mu a}, M_{a b}$ are the generators of the boost transformation along the longitudinal direction, generalized boosts and rotations in the transverse space, and $Z, Z_{\mu}$ are the extended elements. Our conventions for the metric tensor are $\eta_{\mu \nu}=-,+, \eta_{a b}=+,+, \ldots+.[\mu \nu]$ means antisymmetry in the interchange of the two indices $\mu \nu$. The Levi-Civita tensor is defined with $\epsilon^{01}=+1$.

Observe that the commutator of $M_{\mu \nu}$ and $M_{\rho \sigma}$ is zero. This is due to the fact that in 2 dimensions $(\mu, \nu=0,1)$ there is only one generator of Lorentz transformations, and no rotations.

The action of a nonrelativistic string can be obtained by the method of nonlinear realizations [24-26]. Let us consider the coset $G / H$, with the stability group $H$ generated by $\left(M_{\mu \nu}, M_{a b}\right)$.

Locally we parametrize a coset element as [27]

$$
g=e^{i x^{\mu} P_{\mu}} e^{i x^{a} P_{a}} e^{i v^{\mu a} M_{\mu a}} e^{i c^{\mu} Z_{\mu}} e^{i c Z} .
$$

The (Goldstone) coordinates of the coset depend on the parameters $\tau, \sigma$ of the world- sheet. The MC 1-form is given by

$$
\begin{aligned}
\Omega= & -i g^{-1} d g \\
= & L^{\mu} P_{\mu}+L^{a} P_{a}+L^{\mu a} M_{\mu a}+\frac{1}{2} L^{\mu \nu} M_{\mu \nu} \\
& +\frac{1}{2} L^{a b} M_{a b}+L_{z}^{\mu} Z_{\mu}+L_{z} Z,
\end{aligned}
$$

where

$$
\begin{gathered}
L^{\mu}=d x^{\mu}, \quad L^{a}=d x^{a}+d x^{\mu} v_{\mu}^{a}, \quad L^{\mu \nu}=0, \\
L^{\mu a}=d v^{\mu a}, \quad L^{a b}=0, \\
L_{z}^{\mu}=d c^{\mu}+d x^{\nu} \epsilon_{\nu}^{\mu}-v_{. a}^{\mu} d x^{a}-\frac{1}{2} d x^{\nu} v_{\nu a} v^{\mu a}, \\
L_{z}=d c+\frac{1}{2} \epsilon_{\mu \nu} v_{. a}^{\mu} d v^{\nu a} .
\end{gathered}
$$

In the case of a four dimensional space-time the extended Galilei algebra has a further extension. In fact, owing to the existence of the two dimensional Levi-Civita tensor, we can construct a nontrivial closed invariant 2-form $\Omega_{2}^{\mu \nu}$ that transforms as a symmetric tensor of order two under the stability group

$$
\Omega_{2}^{(\mu \nu)}=\epsilon_{a b} L^{(\mu a} L^{\nu) b}=d\left(\epsilon_{a b} v^{\mu a} d v^{\nu b}\right) .
$$

The 1-form $\epsilon_{a b} v^{\mu a} d v^{\nu b}$ is not invariant, therefore the Eilenberg-Chevalley cohomology of degree two is nontrivial [4-6]. This implies the possibility of further extending the algebra. We denote the new generators of the algebra by $\tilde{Z}_{(\mu \nu)}$, where $(\mu \nu)$ means symmetric with respect the interchange of $\mu$ and $\nu$.

The new nonvanishing commutation relations are

$$
\begin{array}{r}
{\left[M_{\mu a}, M_{\nu b}\right]=i \eta_{a b} \epsilon_{\mu \nu} Z+i \epsilon_{a b} \tilde{Z}_{(\mu \nu)},} \\
{\left[\tilde{Z}_{(\mu \nu)}, M_{\rho \sigma}\right]=i \eta_{\nu[\rho} \tilde{Z}_{(\mu \sigma)]}+i \eta_{\mu[\rho} \tilde{Z}_{(\nu \sigma)]} .}
\end{array}
$$

In the new extended algebra the MC 1-form has one more term, given by $\frac{1}{2} \tilde{L}_{z}^{\mu \nu} \tilde{Z}_{(\mu \nu)}$, and the coset element is 


$$
g=e^{i x^{\mu} P_{\mu}} e^{i x^{a} P_{a}} e^{i v^{\mu a} M_{\mu a}} e^{i c^{\mu} Z_{\mu}} e^{i c Z} e^{i \frac{i}{2} \tilde{c}^{(\mu \nu)} \tilde{Z}_{(\mu \nu)},}
$$

where $\tilde{c}^{(\mu \nu)}$ are the group parameters associated to generators $\tilde{Z}_{\mu \nu}$. The new term in the MC 1 -form is given by

$$
\tilde{L}^{(\mu \nu)}=d \tilde{c}^{\mu \nu}+\frac{1}{2} \epsilon_{a b} v^{a(\mu} d v^{\nu) b} .
$$

\section{EXOTIC STRING ACTION}

The ordinary nonrelativistic string action can be written as the pullback on the world sheet with coordinates $(\tau, \sigma)$ of the invariant 2-form [25]

$$
\begin{aligned}
\Omega_{2} & =\epsilon_{\mu \nu} L^{\mu} L_{z}^{\nu} \\
& =\epsilon_{\mu \nu} d x^{\mu}\left(d c^{\nu}-v^{\nu b} d x^{b}+\frac{1}{4} d x^{\nu} v_{\sigma a} v^{\sigma a}\right) .
\end{aligned}
$$

In four dimensions we can also consider a tensor valued 2-form [29]

$$
\Omega_{2}^{\rho \nu \lambda}=L^{\rho} \tilde{L}^{(\lambda \nu)}=d x^{\rho}\left(d \tilde{c}^{(\lambda \nu)}+\frac{1}{2} \epsilon_{a b} v^{(\lambda a} d v^{\nu) b}\right),
$$

which allows us to construct an action for an exotic string. If we eliminate the closed 2-form $d x^{\rho} d \tilde{c}^{\mu \nu}$ and we add the antisymmetric part in $\lambda, \nu$, which is a closed form, we get

$$
\Omega_{2}^{\rho \nu \lambda}=d x^{\rho} v^{\lambda a} d v^{\nu b} \epsilon_{a b} .
$$

Notice that

$$
\Omega_{3}^{\rho \nu \lambda}=d\left(\Omega_{2}^{\rho \nu \lambda}\right)=L^{\rho} L^{\lambda a} L^{b \nu} \epsilon_{a b}
$$

is a closed invariant 3-form. Since $\Omega_{2}^{\rho \nu \lambda}$ is not an invariant 2 -form of the unextended string Galilei algebra we conclude that the Eilenberg-Chevalley cohomology of degree 3 is not trivial.

The string action is obtained by taking a linear combination of the pullback of the previous 2-forms on the world sheet. $T$ is the string tension and $\theta_{\rho \nu \lambda}$ is a spurion tensor field that generalizes the magnetic field appearing in the case of a particle in a magnetic field in $2+1$ dimensions.

$$
\begin{aligned}
S= & T \int d^{2} \sigma \operatorname{det}[e]\left(v^{\mu a} e_{\mu}^{j} \partial_{j} x^{a}+\frac{1}{2} v_{\mu a} v^{\mu a}\right) \\
& +\theta_{\rho \nu \lambda} \int d^{2} \sigma \operatorname{det}[e] \epsilon^{\rho \mu}\left(e_{\mu}^{j} v^{\lambda a} \partial_{j} v^{\nu b} \epsilon^{a b}\right) .
\end{aligned}
$$

Here $\partial_{i}=(\partial / \partial \tau, \partial / \partial \sigma)$. The metric tensor is as before $\eta^{\mu \nu}=(-1,+1)$. The $e_{i}^{\mu}=\partial_{i} x^{\mu}$ are the zweibeine, $e_{\mu}^{j}$ are the inverse zweibeine, and $\operatorname{det}[e]=\operatorname{det}\left[e_{i}^{\mu}\right]$ is the corresponding determinant. Notice that $[T]=\ell^{-2}$ and $[\theta]=$ $\ell^{-1}$. In the previous equation $\theta_{\mu \rho \lambda}$ is a spurion tensor.

If we want to get by double-dimensional reduction the action for the exotic particle [7-16], we have to make a particular choice for the parameters $\theta_{\rho \nu \lambda}$. By performing the dimensional reduction of the exotic term in Eq. (20) we find

$$
\theta_{\rho \nu \lambda} \int d \tau \epsilon^{\rho 0} v^{\lambda a} \partial_{\tau} v^{\nu b} \epsilon_{a b},
$$

to be compared with the exotic term of the action for the particle

$$
\kappa \int d \tau v^{0 a} \partial_{\tau} v^{0 b} \epsilon_{a b}
$$

Therefore the unique choice is to take

$$
\theta_{\rho \nu \lambda}=\theta_{\lambda} \epsilon_{\rho \nu}
$$

with $\theta_{1}=0$. Other possible forms of the spurion field will not be discussed in this paper.

\section{DYNAMICS OF THE EXOTIC STRING}

In this section we will study the dynamics of the exotic string that under dimensional reduction produces the exotic particle. We will use Eq. (23) without the restriction on $\theta_{\mu}$. In the static gauge, where we make the identification $\left(x^{0}, x^{1}\right)=(\tau, \sigma)$, the complete string action becomes

$$
\begin{aligned}
S= & T \int d x^{0} d x^{1}\left(v_{\mu a} \partial^{\mu} x^{a}+\frac{1}{2} v_{\mu a} v^{\mu a}\right) \\
& +\theta^{\lambda} \int d x^{0} d x^{1}\left(\eta^{\mu \nu} \partial_{\nu} v_{\mu b} v_{\lambda a} \epsilon^{a b}\right) .
\end{aligned}
$$

The Lagrangian equations of motion are

$$
\begin{gathered}
T\left(-\partial_{\mu} x_{a}+v_{\mu a}\right)+\theta_{\mu} \partial^{\sigma} v_{\sigma b} \epsilon^{a b}-\theta^{\lambda} \partial_{\mu} v_{\lambda_{b}} \epsilon^{b a}=0, \\
\partial^{\mu} v_{\mu a}=0 .
\end{gathered}
$$

The boundary conditions in general imply

$$
\left.\left(-T v_{1 a} \delta x^{a}+\theta^{\lambda} v_{\lambda a} \epsilon^{a b} \delta v_{1 b}\right)\right|_{0} ^{L}=0,
$$

where $L$ is the length of the string.

These conditions can be satisfied, for example, in the following ways:

(1) By choosing both $x^{a}$ and $v^{\lambda a}$ as periodic functions.

(2) By choosing $x^{a}$ periodic and $v^{\lambda a}$ with fixed values at the boundaries.

(3) By choosing periodic $v^{\lambda a}$ and $x^{a}$ with fixed values at the boundaries.

(4) By choosing both $x^{a}$ and $v^{\lambda a}$ with fixed values at the boundaries.

(5) By choosing $v^{\lambda a}=0$ at the boundaries.

Notice that the cases (1) and (5) correspond to a closed and an open string, respectively (case $\theta_{\mu}=0$ ). Notice too that, for $\theta_{\mu}=0$, one can eliminate the variables $v$, which are essentially the derivative of the variable $x$.

In order to understand which are the physical degrees of freedom of the model and their dynamics we will now study the Hamiltonian formalism. 
Let us start by evaluating the canonical momenta:

$$
\Pi_{\mu a}=\frac{\partial \mathcal{L}}{\partial \dot{\boldsymbol{v}}^{\mu a}}, \quad p_{a}=\frac{\partial \mathcal{L}}{\partial \dot{x}^{a}},
$$

One finds the following primary constraints:

$$
\begin{gathered}
\phi_{a}=\Pi_{0 a}+\theta^{\mu} v_{\mu b} \epsilon^{a b}=0, \quad \chi_{a}=\Pi_{1 a}=0, \\
\xi_{a}=\frac{1}{\sqrt{T}}\left(p_{a}+T v_{0 a}\right)=0,
\end{gathered}
$$

where $\Pi_{\mu a}$ are the momenta conjugated to $v_{\mu a}$ and $P_{a}$ the ones conjugated to $x_{a}$, where, for a generic field $\phi, \dot{\phi}=$ $\partial \phi / \partial t=\partial \phi / \partial x^{0}$, with $x^{0}=t$.

The canonical Hamiltonian density turns out to be

$$
\begin{aligned}
\mathcal{H}_{c}= & p_{a} \dot{x}_{a}+\Pi_{\mu a} \dot{v}^{\mu a}-\mathcal{L} \\
= & \frac{1}{2} T\left(v_{0 a}^{2}-v_{1 a}^{2}\right)-T v_{1 a} x_{a}^{\prime}-\theta^{0} v_{0 a} v_{1 b}^{\prime} \epsilon_{a b} \\
& -\theta^{1} v_{1 a} v_{1 b}^{\prime} \epsilon^{a b},
\end{aligned}
$$

where a prime denotes the derivative with respect to $\sigma$ (or $\left.x_{1}\right)$.

It is easily verified that the following combinations of primary constraints,

$$
K_{a}=\frac{(-1)^{a}}{\sqrt{T}}\left(+\theta_{1} \epsilon_{a b} \xi_{b}+\sqrt{T} \chi_{a}\right)
$$

are first class. By taking the independent combinations

$$
S_{a}=\frac{(-1)^{a}}{z}\left(\sqrt{T} \epsilon_{a b} \xi_{b}-\theta_{1} \chi_{a}\right), \quad z^{2}=T+\theta_{1}^{2},
$$

one sees that the primary constraints separate as the firstclass, $K_{a}$, and the second-class ones $\left(\phi_{a}, S_{a}\right)$.

The matrix of the Poisson brackets among the secondclass constraints, $\left(\phi_{a}, S_{a}\right)$, is given by

$$
\begin{array}{ccccc} 
& \phi_{2} & \phi_{3} & S_{2} & S_{3} \\
\phi_{2} & 0 & -2 \theta^{0} & 0 & z \\
\phi_{3} & 2 \theta^{0} & 0 & z & 0 \\
S_{2} & 0 & -z & 0 & 0 \\
S_{3} & -z & 0 & 0 & 0
\end{array}
$$

The determinant of the $\sigma, \sigma^{\prime}$ independent part of this matrix turns out to be proportional to $z^{4}$. We can now evaluate the Dirac brackets with respect to these constraints. Remember that the general form for the Dirac brackets, given a set $\left(\psi_{\alpha}\right)$ of constraints, with a Poisson bracket matrix given by $C_{\alpha, \beta}$, is

$$
\{A, B\}^{*}=\{A, B\}-\sum_{\alpha, \beta}\left\{A, \psi_{\alpha}\right\} C_{\alpha, \beta}^{-1}\left\{\psi_{\beta}, B\right\} .
$$

Just to give an example, considering the set $\left(\phi_{a}, S_{a}\right)$, we find the following result:

$$
\begin{aligned}
\left\{x_{a}(\sigma, \tau), x_{b}\left(\sigma^{\prime}, \tau\right)\right\}^{*}= & -\frac{2 \theta^{0}}{z^{4}} \epsilon_{a b} \delta\left(\sigma-\sigma^{\prime}\right), \\
\left\{v_{\mu a}(\sigma, \tau), v_{\nu b}\left(\sigma^{\prime}, \tau\right)\right\}^{*}= & -\frac{\theta_{1}}{z^{2}} \epsilon_{a b}\left[\left(\eta_{\mu 0} \eta_{\nu 1}+\eta_{\mu 1} \eta_{\nu 0}\right)\right. \\
& \left.+\frac{2 \theta^{0} \theta^{1}}{z^{2}} \eta_{\mu 1} \eta_{\nu 1}\right] \delta\left(\sigma-\sigma^{\prime}\right), \\
\left\{x_{a}(\sigma, \tau), v_{\mu b}\left(\sigma^{\prime}, \tau\right)\right\}^{*}= & \frac{1}{z^{2}} \delta_{a b}\left[\eta_{\mu 0}+\frac{2 \theta^{0} \theta^{1}}{z^{2}} \eta_{\mu 1}\right] \\
& \times \delta\left(\sigma-\sigma^{\prime}\right) .
\end{aligned}
$$

By requiring the stability of the primary constraints, that is by requiring that the Poisson brackets of the primary constraints with the Hamiltonian be zero, one finds the following secondary constraints:

$$
\lambda_{a}=T\left(v_{1 b}+x_{b}^{\prime}\right) \epsilon_{b a}+\theta^{\mu} \boldsymbol{v}_{\mu a}^{\prime}=0 .
$$

With these two constraints the primary first-class constraints $\left(K_{a}, \lambda_{a}\right)$ become second class. In fact, the Dirac matrix of the constraints,

$$
\begin{array}{ccccc} 
& K_{2} & K_{3} & \lambda_{2} & \lambda_{3} \\
K_{2} & 0 & 0 & 0 & -T \\
K_{3} & 0 & 0 & -T & 0 \\
\lambda_{2} & 0 & T & 0 & -\frac{2 T^{2} \theta^{0} \theta_{1}^{2}}{z^{4}} \times \delta\left(\sigma-\sigma^{\prime}\right), \\
\lambda_{3} & T & 0 & \frac{2 T^{2} \theta^{0} \theta_{1}^{2}}{z^{4}} & 0
\end{array}
$$

has a determinant proportional to $T^{4} \neq 0$. We now iterate the procedure to evaluate the final Dirac brackets, including the new set of second-class constraints. The final result is (we write only the nonvanishing Dirac brackets)

$$
\begin{gathered}
\left\{x_{a}(\sigma, \tau), x_{b}\left(\sigma^{\prime}, \tau\right)\right\}^{* *}=-\frac{2 \theta^{0}}{T^{2}} \epsilon_{a b} \delta\left(\sigma-\sigma^{\prime}\right), \\
\left\{x_{a}(\sigma, \tau), v_{0 b}\left(\sigma^{\prime}, \tau\right)\right\}^{* *}=-\frac{1}{T} \delta_{a b} \delta\left(\sigma-\sigma^{\prime}\right), \\
\left\{x_{a}(\sigma, \tau), p_{b}\left(\sigma^{\prime}, \tau\right)\right\}^{* *}=\delta_{a b} \delta\left(\sigma-\sigma^{\prime}\right), \\
\left\{x_{a}(\sigma, \tau), \Pi_{0 b}\left(\sigma^{\prime}, \tau\right)\right\}^{* *}=\frac{\theta^{0}}{T} \epsilon_{a b} \delta\left(\sigma-\sigma^{\prime}\right),
\end{gathered}
$$

with the $* *$ brackets evaluated as in (34), using the inverse of the matrix (37). At this point we can use the full set of constraints and the final phase space can be taken as $\left(x_{a}, p_{a}\right)$.

Notice that using the constraints $\lambda_{a}$ we can form the combination

$$
\begin{aligned}
v_{12} \lambda_{3}-\lambda_{2} v_{13}= & T v_{1 a}^{2}+T v_{1 a} x_{a}^{\prime}-\theta^{0} v_{0 a}^{\prime} v_{1 b} \epsilon^{a b} \\
& -\theta^{1} v_{1 a} v_{1 b}^{\prime} \epsilon^{a b}=0 .
\end{aligned}
$$

By taking the canonical Hamiltonian [see Eq. (30)]

$$
H_{c}=\int d \sigma \mathcal{H}_{c},
$$

we see that the last three terms of the previous identity coincide with the last three terms in $H_{c}$, except for a sign 
and a total derivative in $\sigma$, containing only the $v^{\mu a}$ variables. This total derivative is zero for periodic boundary conditions and it is a constant for fixed boundary conditions. Therefore, apart from this constant, we get

$$
H_{c}=\frac{T}{2} \int d \sigma\left(v_{0 a}^{2}+v_{1 a}^{2}\right) .
$$

\section{EQUIVALENCE WITH THE FREE NONRELATIVISTIC STRING}

In order to express the Hamiltonian in terms of the independent variables $x^{a}$ and $p_{a}$ one has to solve the constraints $\xi_{a}$ and $\lambda_{a}$. Since these constraints in general depend on $v_{1 a}^{\prime}$, the Hamiltonian density that one gets is generally nonlocal. See the appendix for the expression in terms of $x^{a}, p_{a}$. However, we can show that this nonlocality can be eliminated by a convenient change of variables. Let us start considering the simpler case $\theta_{1}=$ 0 , where the constraints do not depend on $v_{1 a}^{\prime}$. By solving the constraints we get

$$
v_{12}=-x_{2}^{\prime}-\frac{\theta^{0}}{T^{2}} p_{3}^{\prime}, \quad v_{13}=-x_{3}^{\prime}+\frac{\theta^{0}}{T^{2}} p_{2}^{\prime} .
$$

Therefore the Hamiltonian is given by

$$
H_{c}=\int d \sigma\left[\frac{1}{2 T} p_{a}^{2}+\frac{1}{2} \frac{\theta^{02}}{T^{3}} p_{a}^{\prime 2}+\frac{T}{2} x_{a}^{\prime 2}+\frac{\theta^{0}}{T} \epsilon^{a b} x_{a}^{\prime} p_{b}^{\prime}\right] .
$$

From (43) we see that defining

$$
y_{2}=x_{2}+\frac{\theta^{0}}{T^{2}} p_{3}, \quad y_{3}=x_{3}-\frac{\theta^{0}}{T^{2}} p_{2},
$$

the Hamiltonian can be written as

$$
H_{c}=\int d \sigma\left(\frac{p_{a}^{2}}{2 T}+\frac{T}{2} y_{a}^{\prime 2}\right) \text {. }
$$

Since the variables $v_{1 a}$ (and correspondingly $y_{a}$ ) have zero Dirac brackets with themselves, the theory is equivalent to a nonrelativistic free string [30].

Coming back to the action in Eq. (24) for the general case $\theta_{1} \neq 0$, if we integrate by parts the last term (neglecting again the total derivative for the reasons explained above) we get

$$
\begin{aligned}
S= & T \int d x^{0} d x^{1}\left[v_{\mu a}\left(-\partial^{\mu} x^{a}-\frac{\theta^{\lambda}}{T} \partial^{\mu} v_{\lambda b} \epsilon^{b a}\right)\right. \\
& \left.+\frac{1}{2} v_{\mu a} v^{\mu a}\right] .
\end{aligned}
$$

By defining now the new variables

$$
y^{a}=x^{a}-\frac{\theta^{\lambda}}{T} v_{\lambda b} \epsilon^{b a},
$$

we see that the model is equivalent to the free nonrelativistic string [24] also for $\theta^{1} \neq 0$, since the new action coincides with the first term in Eq. (20). Also in this case the Dirac brackets of the $y_{a}^{\prime} s$ vanish making the theory a commutative one.

However, we have to consider the boundary conditions discussed in Sec. IV. When $x_{a}$ and $y_{a}$ satisfy the same boundary conditions, as for instance in cases (1), (4), and (5), there is a complete equivalence of this exotic string with the ordinary one. In cases (2) and (3) the $y_{a}$ variables do not satisfy definite boundary conditions and we lose the equivalence. Notice also that, when the equivalence is realized, the invariance group (in the $y_{a}$ variables) is isomorphic to the extended Galilei group without the extended generator $\tilde{Z}_{(\mu \nu)}$.

\section{DISCUSSION}

In this paper we have constructed an exotic classical nonrelativistic string in $3+1$ dimensions. The model contains, apart from the ordinary nonrelativistic action, an extra term with a spurion field that exists only in $3+1$ dimensions. The existence of this extra term is associated to the existence of a new term in the extended Galilei algebra in 4 dimensions. In general the model is noncommutative in the sense that the Dirac brackets of the physical coordinates are not vanishing. It is possible to introduce a new set of coordinates in which the model becomes commutative if the boundary conditions of the old and the new coordinates coincide. We should point out that the transformation properties of the old and the new coordinates are different with respect to the Galilei transformations.

This exotic string by double-dimensional reduction reproduces the exotic Galilei particle in $2+1$ dimensions.

It should be noticed that the model considered in this paper could be obtained from an exotic relativistic model in 4 dimensions as the nonrelativistic limit.

We conclude by saying that it will be interesting to see if an exotic string theory exists for a nonflat case, in such a way that under double-dimensional reduction it could reproduce the exotic Newton-Hooke particle in $2+1$ dimensions.

\section{ACKNOWLEDGMENTS}

We are grateful to Jaume Gomis and Kiyoshi Kamimura for interesting discussions. This work has been supported by European EC-RTN Project Nos. MRTN-CT-2004005104, MCYT FPA 2004-04582-C02-01, and CIRIT GC 2005SGR-00564. One of us, J. G., would like to thank the Galileo Galilei Institute for Theoretical Physics for its hospitality and INFN for partial support during part of the elaboration of this work.

\section{APPENDIX: THE HAMILTONIAN DENSITY IN THE REDUCED CANONICAL SPACE}

From the constraints $\lambda_{a}$ and $\xi_{a}$ the variables $v_{\mu a}$ can be obtained as a function of the canonical coordinates of the 
reduced space $\left\{x^{a}, p_{a}\right\}$. To this end we must solve the following differential system:

$$
\begin{aligned}
& v_{12}^{\prime}(\sigma)=\omega v_{13}(\sigma)+f_{1}(\sigma), \\
& v_{13}^{\prime}(\sigma)=-\omega v_{12}(\sigma)+f_{2}(\sigma),
\end{aligned}
$$

where

$$
f_{1}=\omega\left(x_{3}^{\prime}-\frac{\theta_{0}}{T^{2}} p_{2}^{\prime}\right), \quad f_{2}=-\omega\left(x_{2}^{\prime}+\frac{\theta_{0}}{T^{2}} p_{3}^{\prime}\right) .
$$

The other variables $v_{0 a}$ are easily obtained from the constraints $\xi_{a}$. In these equations we have put $\omega=T / \theta^{1}$. Observe that $\omega$ becomes infinite when $\theta^{1}$ is zero. In this limit the system degenerates to an algebraic system, which was already discussed in Sec. V. The system (A1) can be easily integrated, and the solution is

$$
v_{12}=\frac{1}{\sqrt{2}}\left(w_{1}+w_{2}\right), \quad v_{13}(\sigma)=\frac{i}{\sqrt{2}}\left(w_{1}-w_{2}\right),
$$

where

$w_{1}(\sigma)=\frac{1}{\sqrt{2}} e^{i \omega \sigma}\left(\int_{0}^{\sigma} e^{-i \omega \sigma^{\prime}}\left(f_{1}\left(\sigma^{\prime}\right)-i f_{2}\left(\sigma^{\prime}\right)\right) d \sigma^{\prime}+d\right)$,

$w_{2}(\sigma)=w_{1}(\sigma)^{*}$.

In these equations the complex constant of integration $d$ is determined by the boundary conditions discussed in Sec. IV. With these expressions one can evaluate the canonical Hamiltonian $H_{c}$ [see Eq. (42)]. The resulting Hamiltonian is manifestly nonlocal in the variables $\left(x^{a}, p_{a}\right)$.
[1] J.-M. Lévy-Leblond, in Group Theory and Applications, edited by Loebl (Acad. Press, New York, 1972), Vol. II, p. 222.

[2] A. Ballesteros, M. Gadella, and M. del Olmo, J. Math. Phys. (N.Y.) 33, 3379 (1992).

[3] Y. Brihaye, C. Gonera, S. Giller, and P. Kosiński, arXiv:hep-th/9503046.

[4] V. Bargmann, Ann. Math. 59, 1 (1954).

[5] J.-M. Lévy-Leblond, Commun. Math. Phys. 12, 64 (1969).

[6] J. A. de Azcarraga and J. M. Izquierdo, Lie Groups, Lie Algebras, Cohomology and some Applications in Physics (Cambridge University Press, Cambridge, England, 1995).

[7] J. Lukierski, P. C. Stichel, and W. J. Zakrzewski, Ann. Phys. (N.Y.) 260, 224 (1997).

[8] C. Duval and P. A. Horvathy, Phys. Lett. B 479, 284 (2000); J. Phys. A 34, 10097 (2001).

[9] R. Jackiw and V. P. Nair, Phys. Lett. B 480, 237 (2000).

[10] C. Duval, Z. Horvath, and P. A. Horvathy, Int. J. Mod. Phys. B 15, 3397 (2001).

[11] P. A. Horvathy and M. S. Plyushchay, J. High Energy Phys. 06 (2002) 033.

[12] P. A. Horvathy and M. S. Plyushchay, Phys. Lett. B 595, 547 (2004).

[13] P. A. Horvathy and M. S. Plyushchay, Nucl. Phys. B714, 269 (2005).

[14] J. Negro, M. A. del Olmo, and J. Tosiek, J. Math. Phys. (N.Y.) 47, 033508 (2006).

[15] M. A. del Olmo and M. S. Plyushchay, Ann. Phys. (N.Y.) 321, 2830 (2006).
[16] P. A. Horvathy, arXiv:cond-mat/0609571.

[17] A. P. Polychronakos, arXiv:0706.1095.

[18] S. Dhar, B. Basu, and S. Ghosh, arXiv:cond-mat/0701096.

[19] O. Arratia, M. A. Martin, and M. A. Olmo, arXiv:math-ph/ 9903013.

[20] P.D. Alvarez, J. Gomis, K. Kamimura, and M.S. Plyushchay, Ann. Phys. (N.Y.) 322, 1556 (2007).

[21] J. Gomis and H. Ooguri, J. Math. Phys. (N.Y.) 42, 3127 (2001).

[22] U.H. Danielsson, A. Guijosa, and M. Kruczenski, J. High Energy Phys. 10 (2000) 020.

[23] S. R. Coleman, J. Wess, and B. Zumino, Phys. Rev. 177, 2239 (1969); C. G. Callan, S. R. Coleman, J. Wess, and B. Zumino, Phys. Rev. 177, 2247 (1969).

[24] J. Brugues, T. Curtright, J. Gomis, and L. Mezincescu, Phys. Lett. B 594, 227 (2004).

[25] J. Brugues, J. Gomis, and K. Kamimura, Phys. Rev. D 73, 085011 (2006).

[26] J. Gomis, K. Kamimura, and P. West, Classical Quantum Gravity 23, 7369 (2006).

[27] The introduction of a Goldstone field associated to a central extension was first considered in [28]

[28] J. P. Gauntlett, J. Gomis, and P. K. Townsend, Phys. Lett. B 249, 255 (1990).

[29] We could have considered also a scalar 2 -form $\tilde{\Omega}=$ $L_{z} \eta_{\mu \nu} \tilde{L}^{(\lambda \nu)}$, however under double-dimensional reduction it does not produce the action of an exotic particle

[30] J. Gomis and F. Passerini, Phys. Lett. B 617, 182 (2005). 